\title{
Enhancement of Token Ring LAN Performance Using Multiple Tokens Technique
}

\author{
Dr. Qutaiba I. Ali \\ Computer Eng. Dept. \\ Mosul University
}

\author{
Dr. A.I.A Jabbar \\ Electrical Eng. Dept. \\ Mosul University
}

\begin{abstract}
Token ring LAN's have been used for many years and occupied an important part of the computer network world. It has the advantage of stabilized performance over variable load conditions. In this paper, a new method is introduced to enhance the characteristics of the LAN. The idea behind the new method is to give the stations on the ring more opportunity to transmit by splitting the main ring into mutiple subrings each one of them use the same protocol of the ordinary token ring LAN and managed by supervisor stations. Discrete event simulation is used to examine the effect of adopting the new method on the performance of the ordinary LAN(for simplicity of simulation, two subrings is considered in this research). The results obtained from the simulation program show the benefit obtained from the new method.
\end{abstract}

Keywords : Token Ring, Throughput, Delay

تحسين أداء شبكة (Token Ring) باستخدام تقنية تعدد الآخذ

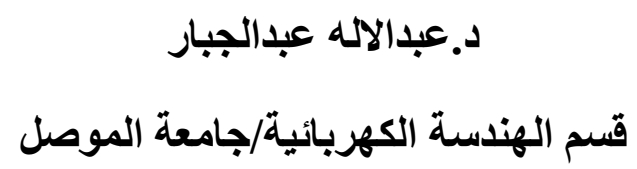

د.قتيبة ابراهيم

قسم هندسة الحاسبات/جامعة الموصل

\section{الخلاصة}

تحتل شبكة Token Ring أهية خاصة في عالم شبكات الحاسب و تمتاز باستقر ارية عالية في

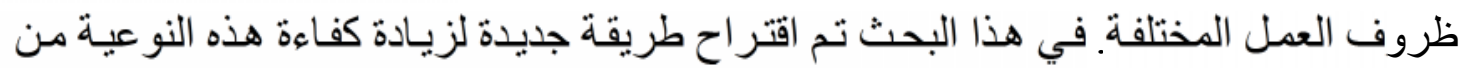

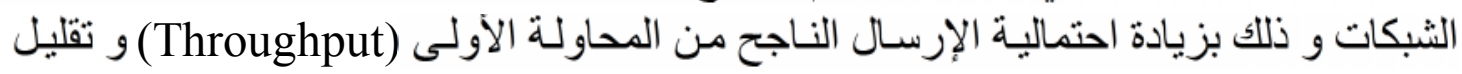


الزمن اللازم لإرسال حزم المعلومات(Delay) و تتلخص هذه الطريقة باختبار محطتين تقومان

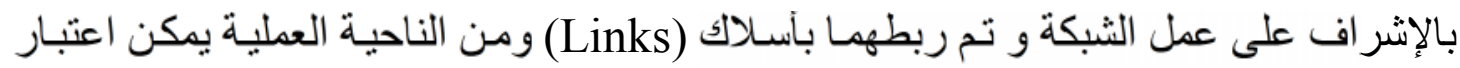

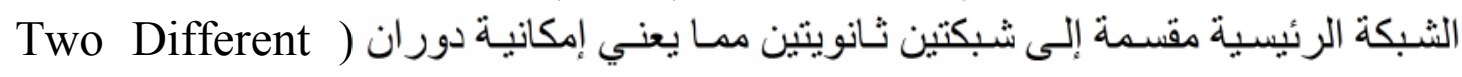

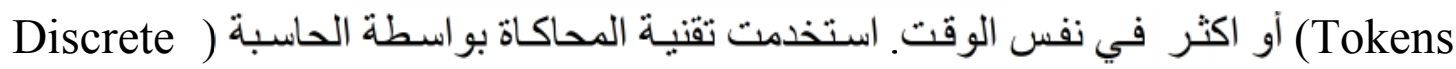
Event Simulation 


\section{Introduction}

Conventional token Ring networks are used primarily in technical and office environments[1-3]. The main principles of operation of these LANs are as follows:

Whenever a station which is initially in the listening mode wants to send a frame, it first waits for the token (a three byte frame, without it no station has the right to send frames through the ring ), then initiating transmission of the data frame after seizing the free token and including it in the header of the frame. The data frame which includes the address of destination is received and then reinserted in the ring bit by bit by all stations in the ring see Fig.( 1 ). This procedure continue until the frame circulates back to the initiating station, which is now considered to be in the transmission mode, where it is either to be saved for comparison with the original data or discarded it. It is obvious that the procedure will provide stations by a copy of the frame, only the desired station takes a copy of the frame while the other will ignore it.

The intended station after receiving the data frame completely sets the response bits at the tail of the data frame as a matter of an acknowledgement. After a successful transmission, the transmitting station releases the seized token. This is done in two ways depending on the bit rate (Speed) of the ring. With slower rings (4 Mbps), the token is released only after the reception of the slotted response bits. With higher speed rings (16 Mbps), it is released after transmitting the last bit of a frame (this is known as early token release) [4-6].

In this paper, a modified token ring operation is adopted to improve the performance of the conventional ring LAN. It is based on splitting the original LAN into multiple sub LAN's (subrings) (for simplicity of simulation, two tokens case is considered in this paper) managed by two supervisor stations. Further details are shown in the next paragraph.

\section{Details of the modified token ring LAN:}

In order to improve the capacity of a token ring LAN to handle extra loads and to limit the effect of a packet delay on the performance of the network, the following modifications are suggested. 
A link as shown in fig ( 2 ) is connected between two supervisor stations(monitoring) which are chosen carefully such that the original token ring LAN is splitted into two subrings with the following conditions :

1. Almost equal numbers of stations are existing in each sub ring to keep equal packet delay to all stations within the original token ring.

2. The ring splitting condition is based on a criteria that, the inter traffic between stations within each subring is higher than the traffic with stations on the other subring.(based on the traffic demands) .

3. Each supervisor station deals with two NIC's to manage the traffic between the two established subrings. See Fig. (2). 


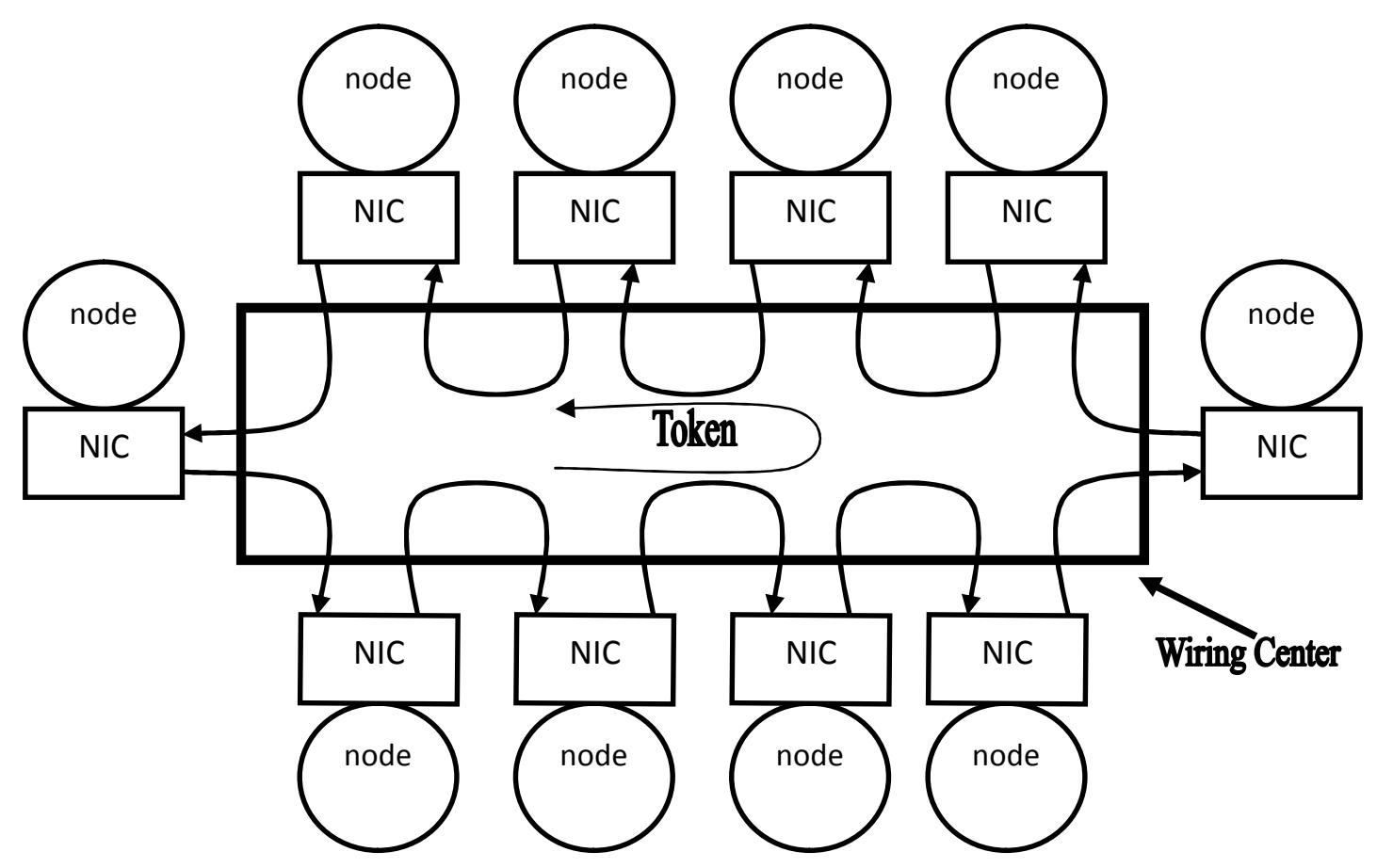

Figure ( 1 ), Token Ring LAN

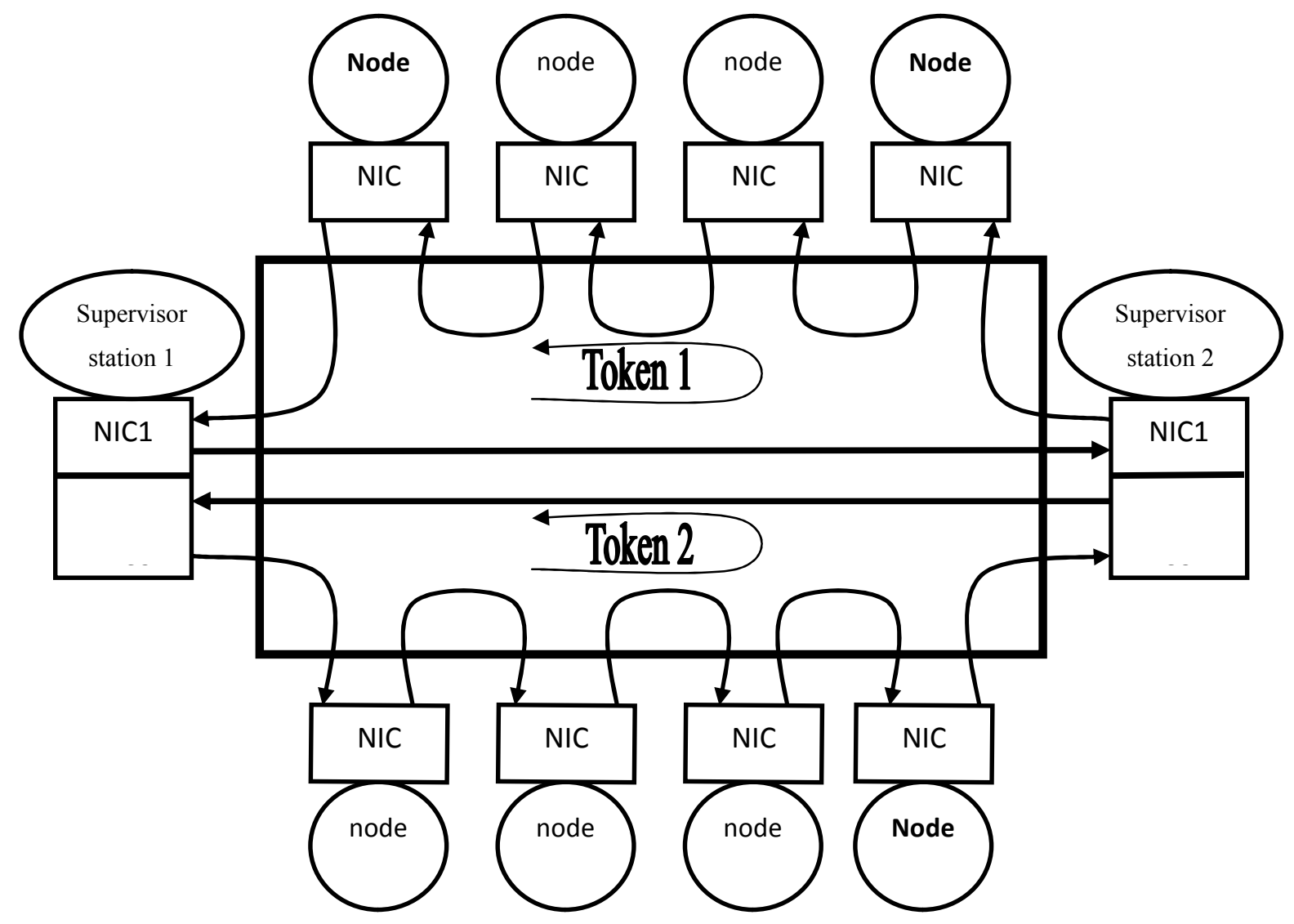

Figure ( 2 ), Structure of the Token Ring LAN 
4. Supervising stations is like any station on the ring but performs transparently additional tasks like for example the management of the traffic on the subrings.

5. Full duplex technique is suggested for the communication between the two supervisor stations through the additional link.

Referring to Fig. (2), if station (A) wishes to transmit a frame to station (B) (on the same subring), it does so using the ordinary token ring protocol algorithm mentioned before. On the other hand, if station (A) wishes to send a frame to station (C) on subring (2), supervisor station (1) has the authority to receive the frame and store it temporarily in its buffers, then acknowledge station (A) for successful transmission. Supervisor station (1), after that waits for a free token coming from subring (2) to complete the transmission of the frame to station (C) (the same procedure is valid for a station on subring (2)trying to transmit to a station on subring(1)).

According to this arrangement, two frames (from different subrings) can be passed simultaneously through the partitioning link, as shown in Fig. (2).

In case of a supervisor station failure, the link between the two supervisor stations is automatically removed and the two subrings joined together to recover the original single ring topology. It is worth while to mention that the remaining supervisor station will introduce a further delay in the ring to compensate the delay of the failed supervisor station (a delay equivalent to 24 bit interval must be available in the ring to keep the capability of the free token to circulate around the ring). If the two supervisor stations failed, the above procedure is still valid and one of the ordinary stations will behave like a supervisor station.

\section{Simulation Model Assumptions:}

The model of the modified token ring is based on the following assumptions:

1. Fixed packet length.

2. Each subring has equal number of stations.

3. Identical Poisson arrival process to each station with mean interarrival time $(\gamma)$ slots (time is divided into slots; each one is equivalent to the maximum propagation delay around the circumstance of the ring).

4. A supervisor station sends all stored packets through the intended ring as soon as it receives a free token. 
5. There is no priority of transmission from a station to another station on the same subring or on the other subring (i.e., equally likely stations).

6. The offered load of each subring is generated from two sources:

a. Stations on the same subring.

b. Few stations on the other subring.

7. Both subrings are almost equal in length.

8. Each subring has its own free token.

9. The token has the standard length of (24) bit.

10.The delay at each subring is enough to keep its own free token circulating around the subring.

11. The latency at each station is equal to (1) bit time (i.e. each station introduces a 1-bit delay in the ring).

12. The performance of the token ring LAN has been validated using the following parameters:

a. Throughput: It is defined as the total number of bits being transferred through the channels successfully from the first try within a given interval of time.

b. Average packet delay: In the conventional token ring simulator, the average packet delay is defined as the total time measured when a packet entered the token ring until it is successfully received.

But in the case of modified token ring simulator it is calculated in the following way:

Average packet delay $=($ Time delay from the source station to supervisor station) + (queuing delay in the supervisor station buffers $)+($ time delay from supervisor station to the destination stations).

\section{The simulation procedure:}

The simulation is based on dividing the stations on each subring into supervisor and background types .The two supervisor stations are common between the two subrings as shown in Fig.( 2 ) with the condition that each supervisor station is responsible about the traffic transferred from a given subring(i.e., supervisor station(1) is responsible about the traffic coming from subring(2) to the environment of subring(1), and supervisor station (2) is responsible about the traffic coming from subring(1) to subring(2)). The simulation is designed such that the calculation of throughput and average packet delay takes into account the interaction between the two types over along interval of time which is divided into a number of 
cycles each one is consisting of a busy and idle periods and each busy period is consisting of a number of slots equal to $(\mathbf{N})$, where:

$\mathrm{N}=$ ((data length (bits)/ (channel capacity (bps)*maximum propagation delay))

Where:

Time of one slot $=$ maximum propagation delay

\section{Procedure algorithm:}

The proposed technique is based on the conventional token ring protocol(i.e., each subring performs the steps required by the token ring protocol. It is based on the following statistical equations:

1. At a given time slot, the number of background stations wishing to transmit follows binomial distribution and it can be calculated using the following eqs. [7]:

$\mathrm{QK}=(\mathrm{Q}-(\mathrm{KB}+\mathrm{KG}))$

$\therefore \mathrm{P}_{\mathrm{to}}=\left(\mathrm{e}^{-\mathrm{T} / \gamma}\right)^{\mathrm{QK}}$

$\mathrm{P}_{\mathrm{t} 1}=\mathrm{P}_{\mathrm{to}}+\left(\mathrm{QK}-\left(1-\mathrm{e}^{-\mathrm{T} / \gamma}\right) \cdot\left(\mathrm{e}^{-\mathrm{T} / \gamma}\right)^{\mathrm{QK}-1}\right)$

$P_{t i}=P_{t i}+\left(\left(Q_{K} ! /\left(Q_{K}-i\right) ! i !\right) \cdot\left(1-e^{-T / \gamma}\right)^{i} \cdot\left(e^{-T / \gamma}\right)^{\mathrm{ek}-1}\right)$

$\mathrm{i}=2,3, \ldots . \mathrm{Q}_{\mathrm{K}}$

Where:

- $\gamma=(\mathrm{L} / \mathrm{B})[\mathrm{L}:$ packet length(Bit), B: offered load percentage]

- $\mathrm{Q}=$ Total number of stations on the ring.

- $(\mathrm{KG})=$ No. of background stations having a packet to be transmitted in the present cycle.

- $(\mathrm{KB})=$ No. of background stations having finished their transmissions and having packets waiting to be transmitted in the next cycle.

- The time interval in the case that the previous cycle is idle and a station received a free token and has no ready packets to be transmitted is equal to :

$\mathrm{T}=$ Token time $+(1)$ bit time $*$ No. of stations + propagation delay between any two stations.

- While the time interval in the case that the previous cycle is busy and a station having ready packet is given by :

$\mathrm{T}=$ Packet length $+(1)$ bit time $*$ No. of stations

The probability $\left(\mathrm{P}_{\mathrm{ti}}\right)$ can be derived as follows: 
With the assumption that the number of packets arrived to the channel follow Poisson distribution, the probability of $(\mathrm{k})$ arrivals is given by:

$\mathrm{P}_{\mathrm{k}}=\left((\mathrm{T} / \gamma)^{\mathrm{k}} / \mathrm{k} !\right) \cdot \mathrm{e}^{-\mathrm{T} / \gamma}$

Where $(T / \gamma)$ is the packets arrival rate (packet/sec.) and $(\gamma)$ is the interarrival time.

The probability of no arrivals is given by:

$\mathrm{P}_{0}=\mathrm{e}^{-\mathrm{T} / \gamma}$

Thus, the probability of at least one of (i) stations having ready packet for transmission:

$\left(1-\mathrm{p}_{0}\right)^{\mathrm{i}}=\left(1-\mathrm{e}^{-\mathrm{T} / \gamma}\right)^{\mathrm{I}}$

The behavior of a group of stations is practically following the classical binomial distribution, which is represented by the general equation:

$P_{i}=(n ! /(n-i) ! i !) \cdot G^{i} \cdot(1-G)^{n-I}$

This equation gives the probability of occurrence of event $(G)$ in an (n) trials for any of (i) sources. Thus, $\left(\mathrm{P}_{\mathrm{i}}\right)$ as illustrated by equation (2) can be obtained simply by replacing $(\mathrm{n})$ by $\left(\mathrm{Q}_{\mathrm{k}}\right)$ and $(\mathrm{G})$ by $\left(1-\mathrm{e}^{-\mathrm{T} / \gamma}\right)^{\mathrm{i}}$.

2. The output of a random number generator (RNG) having uniform distribution with a mean value equal to (1) is taken at a given time slot.

3. If the output of the (RNG) lies between $\left(\mathrm{P}_{\mathrm{ti}}\right)$ and $\left(\mathrm{P}_{\mathrm{ti}+1}\right)$ then there is (i) stations having packets ready to be transmitted, i.e. $K G=K G+i$.

4. When a successful transmission occurs, the number of stations having finished their packets transmission is increased by one.

5. The number of stations trying to retransmit again in the next cycle (after finishing their first transmission) is calculated using the following eqs.:

$$
\begin{aligned}
& \mathrm{q}_{\mathrm{to}}=\left(\mathrm{e}^{-\mathrm{T} / \gamma}\right)^{\mathrm{KB}} \\
& \mathrm{q}_{\mathrm{t} 1}=\mathrm{q}_{\mathrm{to}}+\mathrm{KB} \cdot\left(1-\mathrm{e}^{-\mathrm{T} / \gamma}\right) \cdot\left(\mathrm{e}^{-\mathrm{T} / \gamma}\right)^{\mathrm{KB}-1} \\
& \mathrm{q}_{\mathrm{ti}}=\mathrm{q}_{\mathrm{t} 1}+\left((\mathrm{KB} ! /(\mathrm{KB}-\mathrm{i}) ! \mathrm{i} !) \cdot\left(1-\mathrm{e}^{-\mathrm{T} / \gamma}\right)^{\mathrm{i}} \cdot\left(\mathrm{e}^{-\mathrm{T} / \gamma}\right)^{\mathrm{KB}-1}\right) \\
& \mathrm{i}=2,3, \ldots \mathrm{KB}
\end{aligned}
$$


6. If the output of another uniform random number generator lies between $\left(\mathrm{q}_{\mathrm{ti}}\right)$ and $\left(\mathrm{q}_{\mathrm{ti}+1}\right)$, then there is (i) stations trying to retransmit their packets again.

7. The cumulative number of stations wishing to transmit in the next scanning cycle is equal to the initial value of (KG).

From the simulation point of view, there is a free token arrived at the supervisor station only if the number of stations on a subring trying to transmit become zero $(\mathrm{Kg}=0)$. The simulator checks the buffers of the supervisor station in order to discover its entity to transmit. If the buffers are empty (i.e., packets counter $=0$ ), the supervisor station retransmit the free token again. On the other hand, if there are stored packets in the buffers, then the supervisor station transmits all the packets one after the other then updating the packets and buffers counters which are used to calculate the buffer time delay.

The flow chart shown in Fig. (3) illustrates the performance of the modified token ring simulator.

\section{Results and Discussion:}

As a matter of comparison, the performance of a conventional and modified token ring LAN's with different conditions are studied using simulation technique. The following parameters and variables are suggested during the run of the simulation process: 


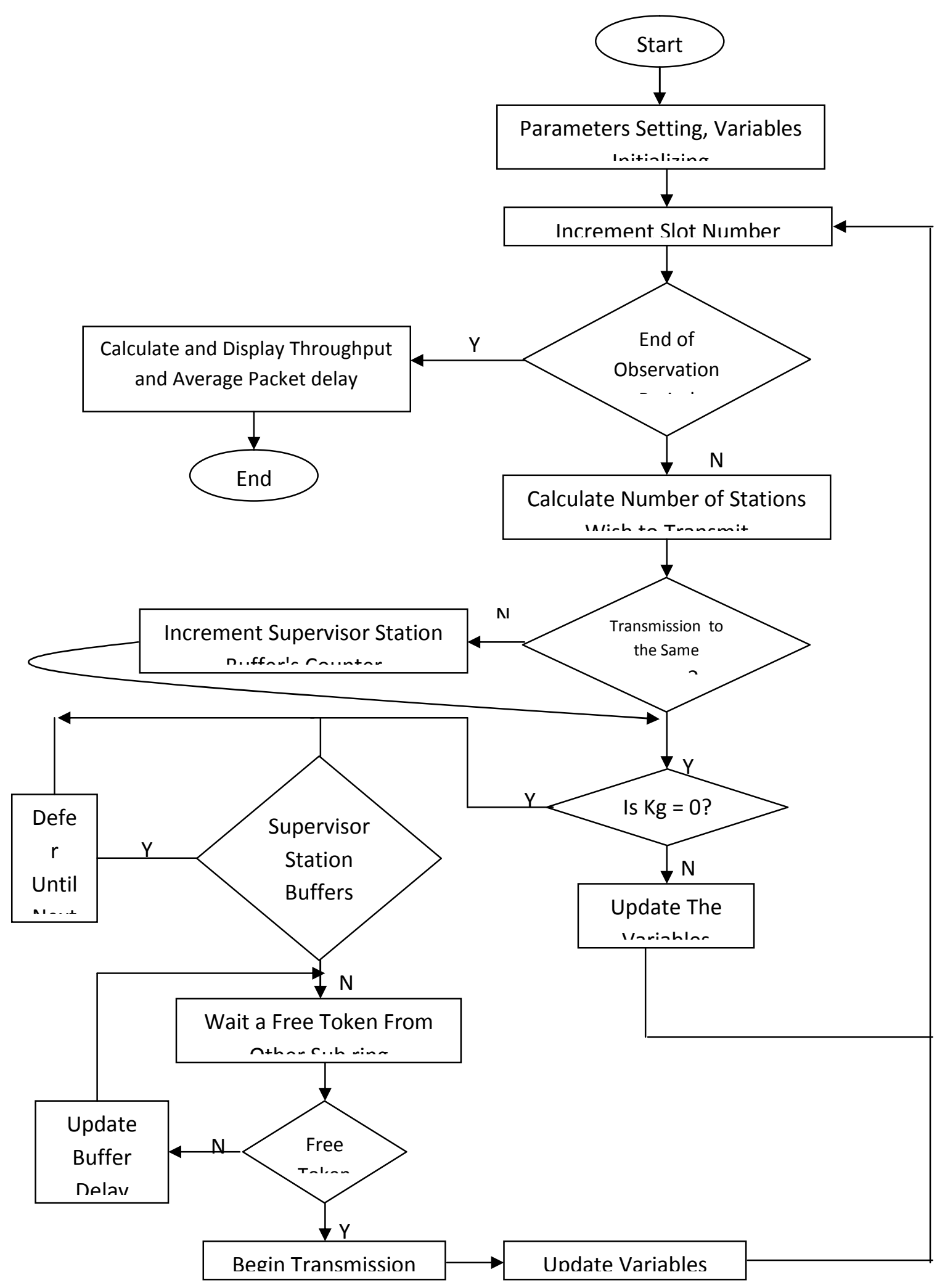

Figure (3), FlowcToken ring simulator 


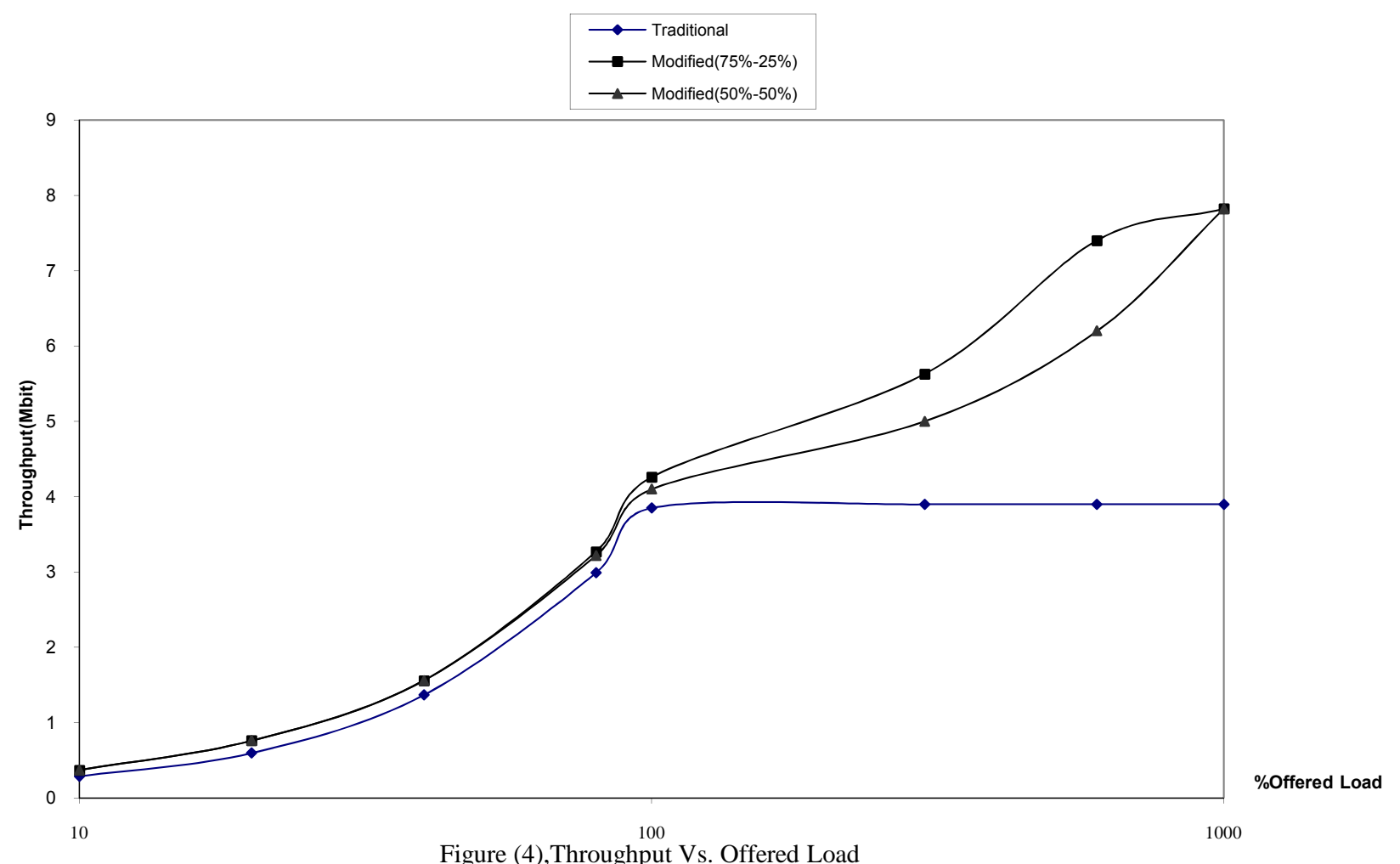

Figure (4),Throughput Vs. Offered Load

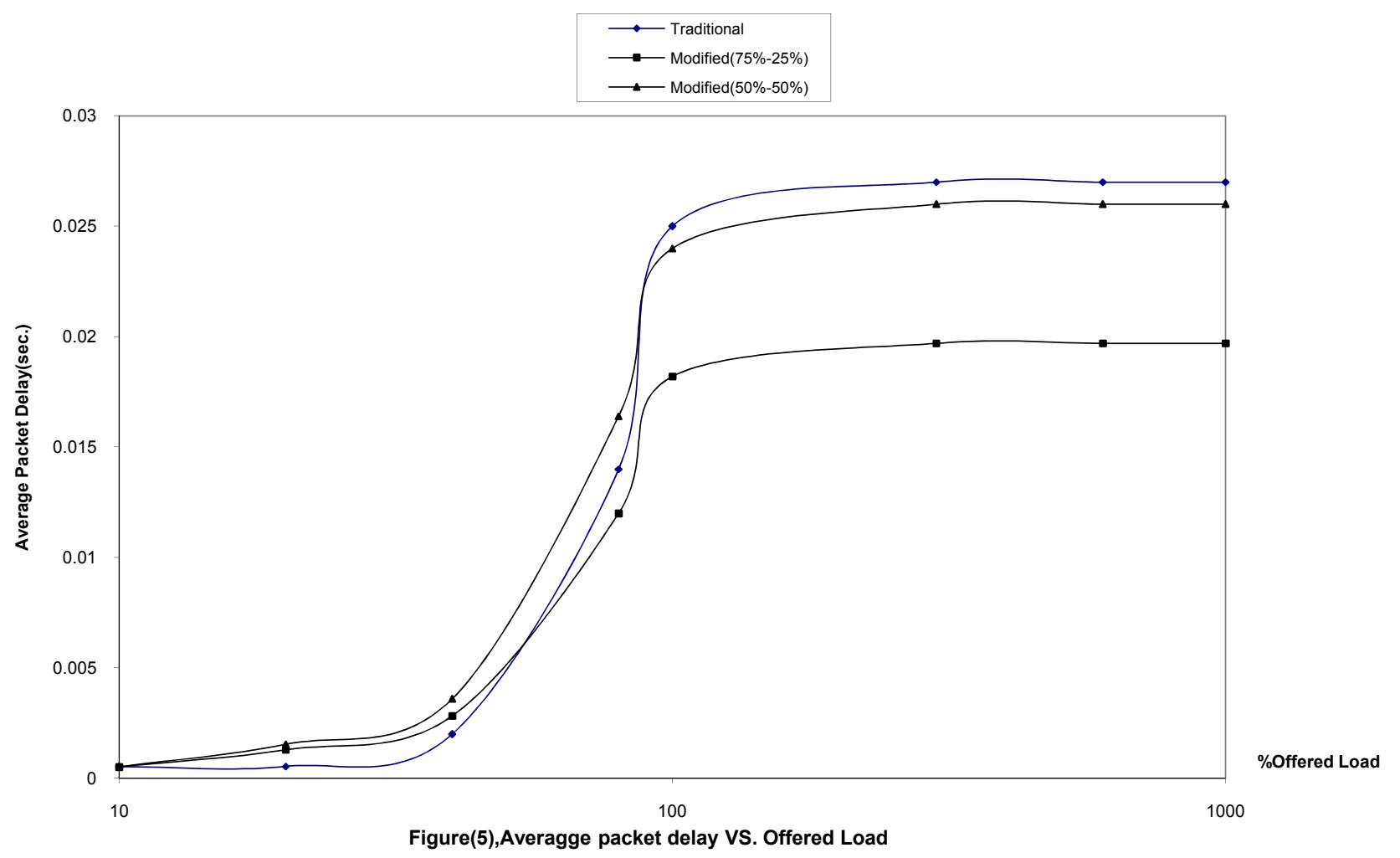


1.Number of stations $=100$ (50 on each subring).

2. Main Ring length $=2(\mathrm{Km})$.

3.data length $=2048$ bit.

4.Bit rate $=4 \mathrm{Mbps}$.

Figures $(4 \& 5)$ shows the throughput and the average packet delay as a function of the offered load(inside subring load percentage, outside subring load percentage), it is obvious that at low offered load, there are small numbers of packets ready to be transmitted, hence, small values of throughput and average packet delay are expected. As the offered load increases, more packets are arrived to the channel, and the busy cycles of the channel increase too, causing an increment in the throughput and a longer average packet delay (a station has to wait longer time before getting a free token ). The deterministic nature of the token ring medium access protocol causes the throughput and the average packet delay to be constant at very high offered load (in such a case, all stations have ready packets for transmission). The figures also indicate that the modified token ring LAN has better performance as compared with the conventional one (especially at high offered load conditions).

The effect of load distribution (the percentage of the load from a subring directed to the other subring) in the modified token ring LAN is studied. Better performance could be obtained if the load circulating in a subring is much greater than the transferred load to the other subring. As a consequence, an increment in the total throughput could be measured due to the simultaneous transmissions on the two subrings and a decrement in the average packet delay due to the shorter path of transmission (on a single subring) as compared with the path around the original token ring LAN.

Confidence about the correctness of the simulation results is ensured, this is achieved by comparing the performance results of a single subring using the modified token ring simulator with that of the ordinary (conventional) token ring simulator on the condition that the working parameters are the same. The following parameters are set to both simulators:

1. (95\% to the same subring-5\% to the other subring) load distribution (in the modified token ring simulator).

2. No. of stations $=50$.

3. data length $=2048$ bit.

4. Bit rate $=4$ Mbps.

5. Ring length $=1000 \mathrm{~m}$. 
The results of the two simulators are shown in Fig's $(6 \& 7)$. The excellent agreement, between them gives an enough justification about the correctness of the modified token ring simulator algorithm in evaluating the new technique. 


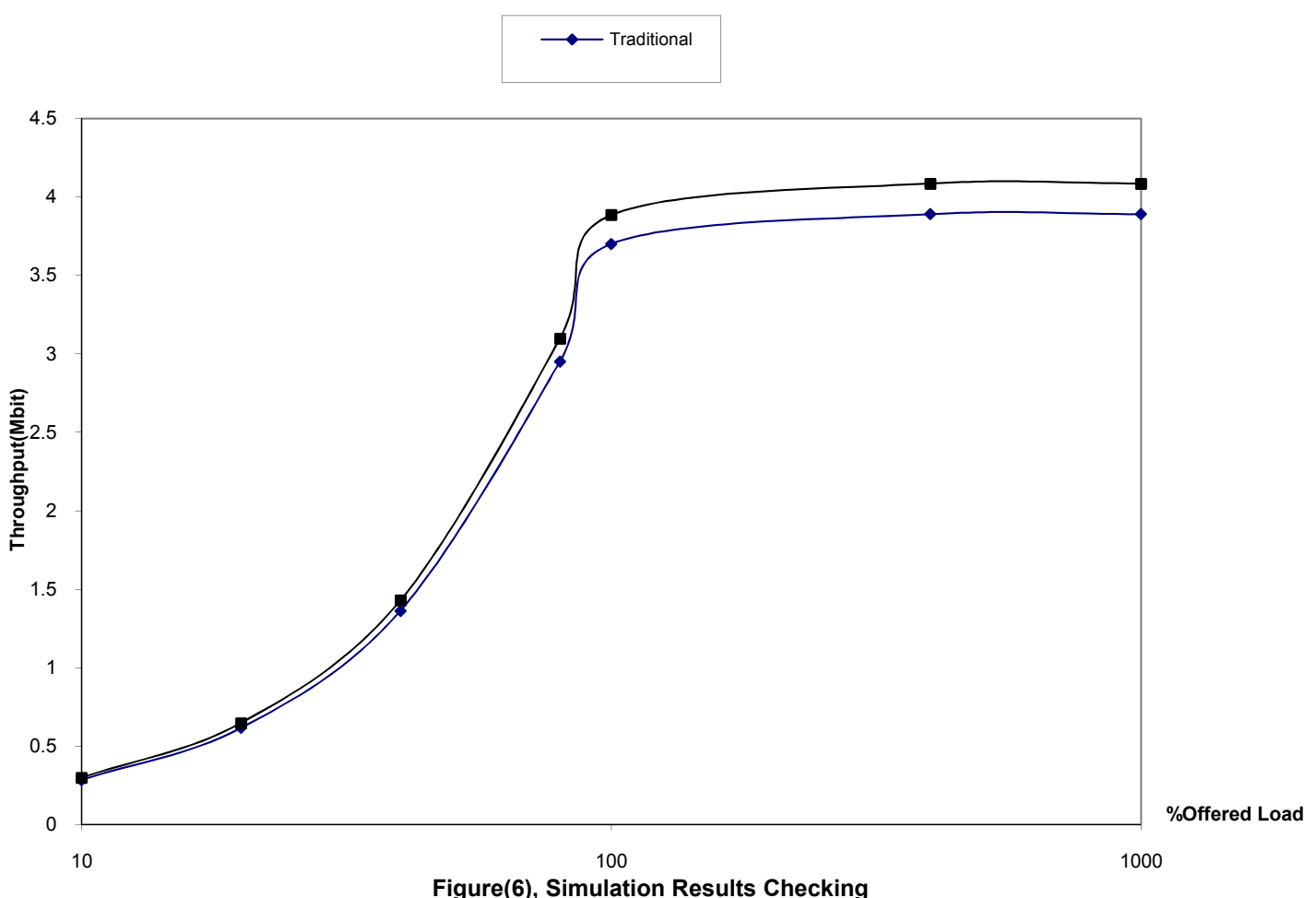

Figure(6), Simulation Results Checking

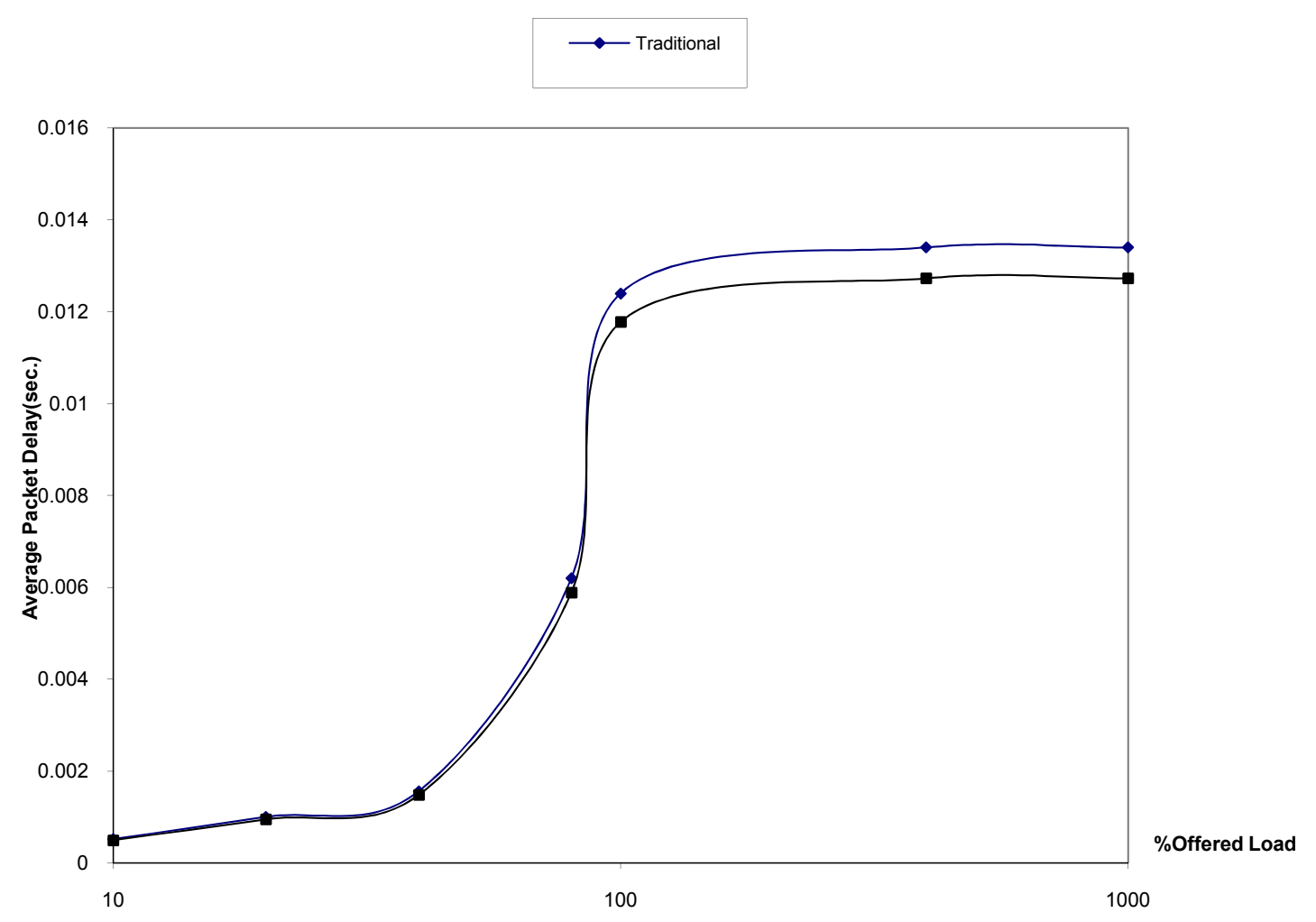

Figure(7), Simulation Results Checking 


\section{Conclusion:}

The novel technique which is introduced in this paper shows a good improvement in the performance of a token ring LAN. It is based on splitting the main ring into two subrings. Accordingly, a station has more chance to transmit its data over the network with lower delay time. As a result of this partitioning scheme and at a given time, two tokens passing through the whole network is some thing normal. Consequently, an improvement in the throughput and delay is expected and the simulation results approved that (an improvement in the delay and throughput performance of about $32 \%$ is obtained). As a suggestion for future work, a dynamic splitting criteria could be applied to determine the best splitting points (which suffers from heavy load) to overcome the problem of increased packet delay. According to this procedure, one of the LAN stations should monitor the traffic load levels on the ring to determine the position of the partition.

\section{References:}

1.Buchanan W. ,'Advanced Data Communication and Networks", Chapman \& Hall Publishing,1997.

2.Forouzan B. ,'Data Communications and Networkings", 4'th Edition, Mcgraw-Hill Publishing , 2006.

3.Keiser G. ,’Local Area Networks”, Mcgraw-Hill Publishing,1989.

4.Tanenbaum A.S. , "Computer Networks",4'th Edition, Prentice-Hall Publishing,2003.

5.Stallings W. , 'Data \& Computer Communications", Sixth Edition , Prentice- Hall Publishing,2003.

6.Halsall F. , "Data Communication, Computer Networks \& Open Systems", Addison-Wesley Publishing, 1996.

7.Ibrahem Q. \& Jabbar A. , “ A simulation Study of Different Local Area Networks", Msc Thesis, Univ. of Mosul , 1998. 
